\title{
Full shifts and irregular sets
}

\section{Luis Barreira}

Departamento de Matemática, Instituto Superior Técnico 1049-001 Lisboa, Portugal

E-mail address: barreira@math.ist.utl.pt

\section{Jinjun Li}

Departamento de Matemática, Instituto Superior Técnico 1049-001 Lisboa, Portugal

Department of Mathematics, Zhangzhou Normal University

Zhangzhou, 363000, P. R. China

E-mail address: 1i-jinjun@163.com

\section{Claudia Valls}

Departamento de Matemática, Instituto Superior Técnico 1049-001 Lisboa, Portugal

E-mail address: cvalls@math.ist.utl.pt

Dedicated to Luís Magalhães and Carlos Rocha on the occasion of their 60th birthdays

\begin{abstract}
By Birkhoff's ergodic theorem, the set of points $X_{\varphi}$ for which the Birkhoff averages of a continuous function $\varphi$ diverge has zero measure with respect to any finite invariant measure. Thus, at least from the point of view of ergodic theory, this set could not be smaller. Nevertheless, it can be large from other points of view. For example, for subshifts with the weak specification property, we showed recently that $X_{\varphi}$ is residual whenever it is nonempty (it is a simple exercise to show that $X_{\varphi}$ is dense whenever it is nonempty). The main purpose of this note is to convey in the simplest possible manner the proof of our result in the particular case of the full shift on a finite number of symbols. This has the advantage of avoiding some accessory technicalities that are necessary in the general case. In fact, we consider also the more general case when the set of accumulation points of the Birkhoff averages of a continuous function is a prescribed closed interval and we show that it is residual whenever it is nonempty.
\end{abstract}

2010 Mathematics Subject Classification. Primary 37B10.

Key words: Full shifts, irregular sets, residual sets. 


\section{Introduction}

We first introduce the notion of the irregular set for the Birkhoff averages of a given function. Given a continuous map $f: X \rightarrow X$ on a compact metric space, the irregular set for the Birkhoff averages of a function $\varphi: X \rightarrow \mathbb{R}$ is defined by

$$
X_{\varphi}=\left\{x \in X: \liminf _{n \rightarrow \infty} \frac{1}{n} \sum_{i=0}^{n-1} \varphi\left(f^{i}(x)\right)<\limsup _{n \rightarrow \infty} \frac{1}{n} \sum_{i=0}^{n-1} \varphi\left(f^{i}(x)\right)\right\} .
$$

As a consequence of Birkhoff's ergodic theorem, the irregular set has zero measure with respect to any finite $f$-invariant measure $\mu$ on $X$ (this means that $\mu\left(f^{-1} A\right)=\mu(A)$ for any measurable set $\left.A \subset X\right)$.

Theorem 1. For a continuous map $f$ on a compact metric space, if the function $\varphi$ is continuous, then $\mu\left(X_{\varphi}\right)=0$ for any $f$-invariant finite measure $\mu$ on $X$.

On the other hand, it was shown in [4] that from the point of view of topological dynamics and dimension theory the set $X_{\varphi}$ can be as large as the whole space. We formulate only a particular case of the results, for the full shift, which is also the dynamical system considered in this note.

Theorem 2. For the full shift $f$ on a finite number of symbols, if the function $\varphi$ is Hölder continuous, then $X_{\varphi}$ is either empty or has Hausdorff dimension equal to the Hausdorff dimension of the whole space $X$.

This phenomenon was first observed by Pesin and Pitskel in [9] for the full shift on two symbols. We refer the reader to the book [1] for a detailed discussion and to $[3,5,6,7,8,10]$ for related work. Besides the Hausdorff dimension one may also consider the topological entropy and more generally the topological pressure of the irregular set.

Here we consider yet another point of view for which an irregular set can be very large, unlike what happens from the point of view of ergodic theory. Namely, for the full shift on a finite number of symbols and for an arbitrary continuous function $\varphi$, we show that the set $X_{\varphi}$ is either empty or residual (we recall that a set is said to be residual if it contains a dense $G_{\delta}$ set).

Theorem 3. For the full shift $f$ on a finite number of symbols, if the function $\varphi$ is continuous, then $X_{\varphi}$ is either empty or residual.

This is a particular case of results of ours in [2] that consider the general class of subshifts with the weak specification property. Roughly speaking, a symbolic system is said to have the weak specification property if under 
iteration one can go from one cylinder set to another (see (1) for the definition) eventually staying outside both of them for a bounded period of time, independently of the initial and final cylinder sets.

In fact, we show in the remainder of the paper that some subsets of the irregular set are also residual. Namely, given an interval $I \subset \mathbb{R}$, let

$$
X_{I}=\left\{x \in X: A_{\varphi}(x)=I\right\},
$$

where $A_{\varphi}(x)$ is the set of accumulation points of the sequence of Birkhoff averages

$$
\frac{1}{n} \sum_{i=0}^{n-1} \varphi\left(f^{i}(x)\right) .
$$

This is the content of Theorem 4, of which Theorem 3 is a corollary. We refer the reader to [2] for details.

In order to show that the set $X_{I}$ is residual we bridge together strings of sufficiently large length corresponding to Birkhoff averages with different limits in the interval $I$. Going back and forth between strings corresponding to these limits one can ensure that the resulting Birkhoff averages diverge and thus their initial points belong to the irregular set. The arguments in [2] are also inspired in this idea, although since we are considering arbitrary subshifts with the weak specification property various technical complications arise that to some extent hide the main idea of the proof.

\section{Formulation of the result}

Let $\sigma$ be the shift map on $\Sigma=\{1, \ldots, k\}^{\mathbb{N}}$, where $k \geq 2$ is an integer. Moreover, let $\varphi: \Sigma \rightarrow \mathbb{R}$ be a continuous function and consider the level sets

where

$$
B_{\varphi}(\alpha)=\left\{\omega \in \Sigma: \lim _{n \rightarrow \infty} S_{\varphi}(\omega, n)=\alpha\right\},
$$

$$
S_{\varphi}(\omega, n)=\frac{1}{n} \sum_{i=0}^{n-1} \varphi\left(\sigma^{i}(\omega)\right) .
$$

We also consider the nonempty closed interval

$$
\mathcal{L}_{\varphi}=\left\{\alpha \in \mathbb{R}: B_{\varphi}(\alpha) \neq \varnothing\right\}
$$

and the set $A_{\varphi}(\omega)$ of accumulation points of the sequence $n \mapsto S_{\varphi}(\omega, n)$.

Theorem 4. Let $\varphi: \Sigma \rightarrow \mathbb{R}$ be a continuous function. Given a closed interval $I \subset \mathcal{L}_{\varphi}$ that is not a singleton, if the set

$$
\Sigma_{\varphi, I}:=\left\{\omega \in \Sigma: A_{\varphi}(\omega)=I\right\}
$$

is nonempty, then it is residual. 
Proof. We first introduce some notation. For $n \in \mathbb{N}$, let $\Sigma^{n}=\{1, \ldots, k\}^{n}$ and $\Sigma^{*}=\bigcup_{n \in \mathbb{N}} \Sigma^{n}$. For each $\omega \in \Sigma^{n}$, we write $|\omega|=n$ and

$$
[\omega]=\{\rho \in \Sigma: \rho \mid n=\omega\},
$$

where

$$
\left(\omega_{1} \cdots\right) \mid n=\left(\omega_{1} \cdots \omega_{n}\right) .
$$

Given $W_{1}, \ldots, W_{n} \subset \Sigma^{*}$ and $\omega \in \Sigma^{*}$, we write

$$
\omega W_{1} \cdots W_{n}=\left\{\omega \omega_{1} \cdots \omega_{n}: \omega_{i} \in W_{i}, 1 \leq i \leq n\right\}
$$

and $W^{\bowtie n}=W_{1} \cdots W_{n}$ when $W_{1}=\cdots=W_{n}=W$.

We proceed with the proof of the theorem. For each $\alpha \in \mathbb{R}, n \in \mathbb{N}$ and $\varepsilon>0$, write

$$
F(\alpha, n, \varepsilon)=\left\{\omega \mid n: \omega \in \Sigma \text { and }\left|S_{\varphi}(\omega, n)-\alpha\right|<\varepsilon\right\} .
$$

Now let $k \in \mathbb{N}$ and choose $\alpha_{k, 1}, \ldots, \alpha_{k, q_{k}} \in I$ such that

$$
I \subset \bigcup_{i=1}^{q_{k}} B\left(\alpha_{k, i}, 1 / k\right)
$$

and

$$
\left|\alpha_{k, i+1}-\alpha_{k, i}\right|<\frac{1}{k} \text { for } i=0, \ldots, q_{k}-1, \quad\left|\alpha_{k, q_{k}}-\alpha_{k+1,1}\right|<\frac{1}{k} .
$$

Moreover, let $\varepsilon_{1}>\varepsilon_{2}>\cdots$ be a sequence of positive numbers decreasing to zero and let

$$
n_{1,1}<n_{1,2}<\cdots<n_{1, q_{1}}<n_{2,1}<n_{2,2}<\cdots<n_{2, q_{2}}<\cdots
$$

be a sequence of positive integers such that

$$
F\left(\alpha_{k, i}, n_{k, i}, \varepsilon_{k}\right) \neq \varnothing \quad \text { for } \quad k \in \mathbb{N}, 1 \leq i \leq q_{k} .
$$

It follows from Birkhoff's ergodic theorem that this choice can be made.

Let $\Omega_{0} \in \bigcup_{n \in \mathbb{N}} \Sigma^{n}$. For each $\omega \in \Omega_{0}$, we choose integers $\left\{N_{k, i}\right\}_{k \in \mathbb{N}, i=1, \ldots, q_{k}}$ (depending on $\omega$ ) such that:

(i) $N_{1, i} \geq 2^{n_{1, i+1}}$ for $2 \leq i \leq q_{1}-1$,

$N_{k, i} \geq 2^{n_{k, i+1}}$ for $k \geq 2,1 \leq i \leq q_{k}-1$,

$N_{k, q_{k}} \geq 2^{n_{k+1,1}}$ for $k \geq 1$;

(ii) $N_{k, i+1} \geq 2^{|\omega|+N_{1,1} n_{1,1}+N_{1,2} n_{1,2}+\cdots+N_{k, i} n_{k, i}}$,

$N_{k+1,1} \geq 2^{|\omega|+N_{1,1} n_{1,1}+N_{1,2} n_{1,2}+\cdots+N_{k, q_{k}} n_{k, q_{k}} \text { for } k \in \mathbb{N}, 1 \leq i \leq q_{k}-1 .}$ 
Moreover, we define sets $\Omega_{k, i} \subset \Sigma^{*}$ for $k \in \mathbb{N}$ and $i=1, \ldots, q_{k}$ by

$$
\begin{aligned}
\Omega_{1,1} & =\bigcup_{\omega \in \Omega_{0}} \omega F\left(\alpha_{1,1}, n_{1,1}, \varepsilon_{1}\right)^{\bowtie N_{1,1},} \\
\Omega_{1,2} & =\bigcup_{\eta \in \Omega_{1,1}} \eta F\left(\alpha_{1,2}, n_{1,2}, \varepsilon_{1}\right)^{\bowtie N_{1,2},} \\
& \cdots \\
\Omega_{2,1} & =\bigcup_{\eta \in \Omega_{1, q_{1}}} \eta F\left(\alpha_{2,1}, n_{2,1}, \varepsilon_{2}\right)^{\bowtie N_{2,1},}
\end{aligned}
$$

and so on. Finally, let

$$
E_{k, i}=\bigcup_{\omega \in \Omega_{k, i}}[\omega] \text { and } E=\bigcap_{k=1}^{\infty} \bigcap_{i=1}^{q_{k}} E_{k, i} .
$$

Clearly, $E$ is a $G_{\delta}$ set since each cylinder set $[\omega]$ is open. Moreover, by construction, each set $E_{k, i}$ is dense and so it follows from Baire's theorem that $E$ is also dense.

It remains to show that $E \subset \Sigma_{\varphi, I}$ (since then $\Sigma_{\varphi, I}$ contains the dense $G_{\delta}$ set $E$ and hence is residual). We must prove that $A_{\varphi}(\omega)=I$ for $\omega \in E$. We recall that for each $\omega \in E$, there exists $\omega^{0} \in \Omega_{0}$ such that

$$
\omega \in \omega^{0} F\left(\alpha_{1,1}, n_{1,1}, \varepsilon_{1}\right)^{\bowtie N_{1,1}} \ldots .
$$

We first show that $I \subset A_{\varphi}(\omega)$. Given

$$
\alpha \in I \subset \bigcup_{i=1}^{q_{k}} B\left(\alpha_{k, i}, 1 / k\right),
$$

take $i_{k} \in\left\{1, \ldots, q_{k}\right\}$ such that $\alpha \in B\left(\alpha_{k, i_{k}}, 1 / k\right)$. For simplicity of the exposition, we assume that $i_{k} \notin\left\{1, q_{k}\right\}$. Let

$$
s_{r_{k}}=\left|\omega^{0}\right|+\sum_{j=1}^{q_{1}} N_{1, j} n_{1, j}+\cdots+\sum_{j=1}^{i_{k}} N_{k, j} n_{k, j},
$$

where $r_{k}=\left(k, i_{k}\right)$. We will prove that

$$
\left|S_{\varphi}\left(\omega, s_{r_{k}}\right)-\alpha_{r_{k}}\right| \rightarrow 0 \quad \text { when } \quad k \rightarrow \infty
$$

It follows from (6) that

$$
\begin{aligned}
\left|S_{\varphi}\left(\omega, s_{r_{k}}\right)-\alpha\right| & \leq\left|S_{\varphi}\left(\omega, s_{r_{k}}\right)-\alpha_{r_{k}}\right|+\left|\alpha_{r_{k}}-\alpha\right| \\
& <\left|S_{\varphi}\left(\omega, s_{r_{k}}\right)-\alpha_{r_{k}}\right|+\frac{1}{k} \rightarrow 0
\end{aligned}
$$


when $k \rightarrow \infty$. Therefore, $\alpha \in A_{\varphi}(\omega)$ and $I \subset A_{\varphi}(\omega)$. In order to prove (6), write

Since

$$
s_{r_{k}}=\widetilde{s}_{r_{k}}+N_{r_{k}} n_{r_{k}} .
$$

we have

$$
\left|\alpha_{r_{k}}\right| \leq\|\varphi\|:=\max _{\omega \in \Sigma}|\varphi(\omega)|
$$

$$
\begin{aligned}
& \left|\sum_{i=0}^{s_{r_{k}}-1} \varphi\left(\sigma^{i}(\omega)\right)-s_{r_{k}} \alpha_{r_{k}}\right| \\
& \leq\left|\sum_{i=0}^{\widetilde{s}_{r_{k}}-1} \varphi\left(\sigma^{i}(\omega)\right)-\widetilde{s}_{r_{k}} \alpha_{r_{k}}\right|+\left|\sum_{i=\widetilde{s}_{r_{k}}}^{s_{r_{k}}-1} \varphi\left(\sigma^{i}(\omega)\right)-N_{r_{k}} n_{r_{k}} \alpha_{r_{k}}\right| \\
& \leq 2 \widetilde{s}_{r_{k}}\|\varphi\|+\sum_{q=0}^{N_{r_{k}}-1}\left|\sum_{j=0}^{n_{r_{k}}-1} \varphi\left(\sigma^{j}\left(\sigma^{\widetilde{s}_{r_{k}}+q n_{r_{k}}}(\omega)\right)\right)-n_{r_{k}} \alpha_{r_{k}}\right| .
\end{aligned}
$$

Now we consider the numbers

$$
v_{n}(\varphi)=\sup \left\{\left|\varphi(\omega)-\varphi\left(\omega^{\prime}\right)\right|: \omega, \omega^{\prime} \in \Sigma, \omega\left|n=\omega^{\prime}\right| n\right\}
$$

and

$$
V_{n}(\varphi)=\sum_{j=1}^{n} v_{j}(\varphi)
$$

By (4) and the definition of the set $F\left(\alpha_{r_{k}}, n_{r_{k}}, \varepsilon_{k}\right)$, one can choose sequences $\bar{\omega}^{0}, \ldots, \bar{\omega}^{N_{r_{k}}-1} \in \Sigma$ such that

$$
\sigma^{\widetilde{s}_{r_{k}}+q n_{r_{k}}}(\omega)\left|n_{r_{k}}=\bar{\omega}^{q}\right| n_{r_{k}}
$$

and

$$
\left|S_{\varphi}\left(\bar{\omega}^{q}, n_{r_{k}}\right)-\alpha_{r_{k}}\right|<\varepsilon_{k}
$$

for $q=0, \ldots N_{r_{k}}-1$. Denoting the last absolute value in (8) by $C_{q}$, it follows from (9) and (10) that

$$
\begin{aligned}
\frac{C_{q}}{n_{r_{k}}} & \leq\left|S_{\varphi}\left(\sigma^{\widetilde{s}_{r_{k}}+q n_{r_{k}}}(\omega), n_{r_{k}}\right)-S_{\varphi}\left(\bar{\omega}^{q}, n_{r_{k}}\right)\right|+\left|S_{\varphi}\left(\bar{\omega}^{q}, n_{r_{k}}\right)-\alpha_{r_{k}}\right| \\
& \leq \frac{V_{n_{r_{k}}}}{n_{r_{k}}}+\varepsilon_{k}
\end{aligned}
$$

for $q=0, \ldots N_{r_{k}}-1$. Together with (8) this implies that

$$
\left|\sum_{i=0}^{s_{r_{k}}-1} \varphi\left(\sigma^{i}(\omega)\right)-s_{r_{k}} \alpha_{r_{k}}\right| \leq 2 \widetilde{s}_{r_{k}}\|\varphi\|+N_{r_{k}}\left(V_{n_{r_{k}}}(\varphi)+n_{r_{k}} \varepsilon_{k}\right) .
$$

São Paulo J.Math.Sci. 6, 2 (2012), 135-143 
Now we observe that it follows from condition (ii) that $\widetilde{s}_{r_{k}} / s_{r_{k}}$ tends to zero when $k \rightarrow \infty$. Indeed, using (5), (7) and condition (ii), we have

$$
\frac{s_{r_{k}}}{\widetilde{s}_{r_{k}}}-1=\frac{N_{r_{k}}}{\widetilde{s}_{r_{k}}} n_{r_{k}} \geq \frac{2^{\widetilde{s}_{r_{k}}}}{\widetilde{s}_{r_{k}}} n_{r_{k}}
$$

and thus, $s_{r_{k}} / \widetilde{s}_{r_{k}} \rightarrow+\infty$ when $k \rightarrow \infty$. Moreover, it follows from the uniform continuity of $\varphi$ that $v_{n}(\varphi) \rightarrow 0$ when $n \rightarrow \infty$. Hence, $V_{n}(\varphi) / n \rightarrow 0$ when $n \rightarrow \infty$ and

$$
\frac{N_{r_{k}} V_{n_{r_{k}}}(\varphi)}{s_{r_{k}}} \leq \frac{V_{n_{r_{k}}}(\varphi)}{n_{r_{k}}} \rightarrow 0 \quad \text { when } \quad k \rightarrow \infty .
$$

By the definition of $s_{r_{k}}$ (see (7)), we have $s_{r_{k}}>N_{r_{k}} n_{r_{k}}$ and $N_{r_{k}} / s_{r_{k}}<$ $1 / n_{r_{k}}$. Therefore,

$$
\left|S_{\varphi}\left(\omega, s_{r_{k}}\right)-\alpha_{r_{k}}\right|<\frac{2 \widetilde{s}_{r_{k}}\|\varphi\|}{s_{r_{k}}}+\frac{V_{n_{r_{k}}}(\varphi)}{n_{r_{k}}}+\varepsilon_{k} \rightarrow 0
$$

when $k \rightarrow \infty$, which completes the proof of (6).

Now we show that $A_{\varphi}(\omega) \subset I$. For each positive integer $n>\left|\omega^{0}\right|$ there exist $k \in \mathbb{N}, i_{k} \in\left\{1,2, \ldots, q_{k}\right\}$ and $0 \leq p<N_{k, i_{k}+1}$ such that

$$
s_{r_{k}}+p n_{t_{k}}<n \leq s_{r_{k}}+(p+1) n_{t_{k}},
$$

where $t_{k}=\left(k, i_{k}+1\right)$. Notice that $k \rightarrow \infty$ when $n \rightarrow \infty$. We claim that

$$
\left|S_{\varphi}(\omega, n)-\alpha_{r_{k}}\right| \rightarrow 0 \quad \text { when } n \rightarrow \infty .
$$

For simplicity of the notation, in a similar manner to that in the former inclusion we assume that $i_{k} \neq q_{k}$. If (13) holds, then it follows from (2) that

$$
\operatorname{dist}\left(S_{\varphi}(\omega, n), I\right) \leq\left|S_{\varphi}(\omega, n)-\alpha_{r_{k}}\right|+\operatorname{dist}\left(\alpha_{r_{k}}, I\right) \rightarrow 0
$$

when $k \rightarrow \infty$. Since $I$ is closed, we conclude that $A_{\varphi}(\omega) \subset I$.

Now we establish property (13). We have

$$
\begin{aligned}
\left|\sum_{i=0}^{n-1} \varphi\left(\sigma^{i}(\omega)\right)-n \alpha_{r_{k}}\right| \leq & \left|\sum_{i=0}^{s_{r_{k}}-1} \varphi\left(\sigma^{i}(\omega)\right)-s_{r_{k}} \alpha_{r_{k}}\right| \\
& +\left|\sum_{i=s_{r_{k}}}^{s_{r_{k}}+p n_{t_{k}}-1} \varphi\left(\sigma^{i}(\omega)\right)-p n_{t_{k}} \alpha_{r_{k}}\right| \\
& +\left|\sum_{i=s_{r_{k}}+p n_{t_{k}}}^{n-1} \varphi\left(\sigma^{i}(\omega)\right)-\left(n-s_{r_{k}}-p n_{t_{k}}\right) \alpha_{r_{k}}\right|
\end{aligned}
$$


and we denote the last two absolute values in (14) respectively by $G_{n}$ and $H_{n}$. We shall estimate each of these terms. In a similar manner to that in (9) and (10), one can choose $\bar{\omega}^{0}, \ldots, \bar{\omega}^{p-1} \in \Sigma$ such that

$$
\sigma^{s_{r_{k}}}+q n_{t_{k}}(\omega)\left|n_{t_{k}}=\bar{\omega}^{q}\right| n_{t_{k}}
$$

and

$$
\left|S_{\varphi}\left(\bar{\omega}^{q}, n_{t_{k}}\right)-\alpha_{t_{k}}\right|<\varepsilon_{k}
$$

for $q=0, \ldots p-1$. Proceeding as in (11), it follows from (3), (15) and (16) that

$$
\begin{aligned}
\left|S_{\varphi}\left(\sigma^{s_{r_{k}}+q n_{t_{k}}}(\omega), n_{t_{k}}\right)-\alpha_{r_{k}}\right| & \leq\left|S_{\varphi}\left(\sigma^{s_{r_{k}}+q n_{t_{k}}}(\omega), n_{t_{k}}\right)-\alpha_{t_{k}}\right|+\left|\alpha_{t_{k}}-\alpha_{r_{k}}\right| \\
& \leq \frac{V_{n_{t_{k}}}(\varphi)}{n_{t_{k}}}+\varepsilon_{k}+\frac{1}{k}
\end{aligned}
$$

for $q=0, \ldots, p-1$. Therefore,

$$
\begin{aligned}
\frac{G_{n}}{n_{t_{k}}} & \leq \sum_{q=0}^{p-1}\left|S_{\varphi}\left(\sigma^{s_{r_{k}}+q n_{t_{k}}}(\omega), n_{t_{k}}\right)-\alpha_{r_{k}}\right| \\
& \leq p\left(\frac{V_{n_{t_{k}}}(\varphi)}{n_{t_{k}}}+\varepsilon_{k}+\frac{1}{k}\right) .
\end{aligned}
$$

Moreover, by (12), we have

$$
H_{n} \leq 2\left(n-s_{r_{k}}-p n_{t_{k}}\right)\|\varphi\| \leq 2 n_{t_{k}}\|\varphi\| .
$$

Collecting the estimates (17) and (18), we obtain

$$
\begin{aligned}
\left|S_{\varphi}(\omega, n)-\alpha_{r_{k}}\right| \leq & \left|S_{\varphi}\left(\omega, s_{r_{k}}\right)-\alpha_{r_{k}}\right|+\frac{2 n_{t_{k}}\|\varphi\|}{n} \\
& +\frac{p\left(V_{n_{t_{k}}}(\varphi)+n_{t_{k}} \varepsilon_{k}\right)}{n}+\frac{p n_{t_{k}}}{k n} .
\end{aligned}
$$

In a similar manner to that in the proof of (6), one can show that the first term in (19) tends to zero when $n \rightarrow \infty$. Moreover, using (12) and condition (i), we obtain

$$
\frac{2 n_{t_{k}}\|\varphi\|}{n} \leq \frac{2 n_{t_{k}}\|\varphi\|}{s_{r_{k}}} \leq \frac{2 n_{t_{k}}\|\varphi\|}{N_{r_{k}}} \rightarrow 0
$$

when $n \rightarrow \infty$. On the other hand, it follows from (12) that

and

$$
\frac{p n_{t_{k}}}{k n} \leq \frac{1}{k} \rightarrow 0
$$

$$
\frac{p\left(V_{t_{k}}(\varphi)+n_{t_{k}} \varepsilon_{k}\right)}{n} \leq \frac{V_{n_{t_{k}}}(\varphi)+n_{t_{k}} \varepsilon_{k}}{n_{t_{k}}} \rightarrow 0
$$


when $n \rightarrow \infty$ (since $k \rightarrow \infty$ when $n \rightarrow \infty$ ). Hence, property (13) follows readily from (19), (20), (22) and (21). This completes the proof of the theorem.

\section{Acknowledgments}

Research partially supported by FCT (grant PTDC/MAT/117106/2010 and CAMGSD). Jinjun Li was also supported by the National Natural Science Foundation of China (Grant No. 11071082) and the Education Committee of Fujian Province (Grant No. JA11173).

\section{References}

1. L. Barreira, Dimension and Recurrence in Hyperbolic Dynamics, Progress in Mathematics 272, Birkhäuser, 2008.

2. L. Barreira, J. Li and C. Valls, Irregular sets are residual, preprint.

3. L. Barreira, B. Saussol and J. Schmeling, Distribution of frequencies of digits via multifractal analysis, J. Number Theory 97 (2002), 410-438.

4. L. Barreira and J. Schmeling, Sets of "non-typical" points have full topological entropy and full Hausdorff dimension, Israel J. Math. 116 (2000), 29-70.

5. E. Chen, T. Küpper and L. Shu, Topological entropy for divergence points, Ergod. Theory. Dynam. Systems 25 (2005), 1173-1208.

6. A.-H. Fan, D.-J. Feng and J. Wu, Recurrence, dimension and entropy, J. London Math. Soc. (2) 64 (2001), 229-244.

7. D.-J. Feng, K.-S. Lau and J. Wu, Ergodic limits on the conformal repellers, Adv. Math. 169 (2002), 58-91.

8. J. Li and M. Wu, Divergence points in systems satisfying the specification property, Discrete Contin. Dyn. Syst. 33 (2013), 905-920.

9. Ya. Pesin and B. Pitskel, Topological pressure and variational principle for noncompact sets, Functional Anal. Appl. 18 (1984), 307-318.

10. D. Thompson, The irregular set for maps with the specification property has full topological pressure, Dyn. Syst. 25 (2010), 25-51. 